\title{
Combination of Intraoperative 5-Aminolevulinic Acid-Induced Fluorescence and 3-D MR Imaging for Guidance of Robotic Laser Ablation for Precision Neurosurgery
}

\author{
Hongen Liao ${ }^{1,2}$, Koji Shimaya ${ }^{1}$, Kaimeng Wang ${ }^{1}$, Takashi Maruyama ${ }^{3}$, \\ Masafumi Noguchi ${ }^{1}$, Yoshihiro Muragaki ${ }^{3}$, Etsuko Kobayashi ${ }^{1}$, Hiroshi Iseki ${ }^{3}$, \\ and Ichiro Sakuma ${ }^{1}$ \\ ${ }^{1}$ Graduate School of Engineering, the University of Tokyo \\ ${ }^{2}$ Translational Systems Biology and Medicine Initiative, The University of Tokyo \\ 7-3-1 Hongo, Bunkyo-ku, Tokyo 113-8656, Japan \\ ${ }^{3}$ Graduate School of Medicine, Tokyo Women's Medical University \\ 8-1 Kawada-cho, Shinjuku-ku, Tokyo 162-8666, Japan \\ liao@bmpe.t.u-tokyo.ac.jp
}

\begin{abstract}
A combination of 5-aminolevulinic acid (5-ALA)-induced fluorescence and three-dimensional (3-D) magnetic resonance imaging (MRI) of a brain tumor has been incorporated into a robotic laser ablation neurosurgery system. 5-ALA is a non-fluorescent prodrug that leads to intracellular accumulation of fluorescent protoporphyrins IX (PpIX) in malignant glioma. The PpIX tends to accumulate in pathological lesions, and emits red fluorescence when excited by blue light. This fluorescence is illuminated with laser excitation, enables intra-operative identification of the position of a tumor and provides guidance for resection with laser photocoagulation. The information provided by the MRI is enhanced by the 5-ALA fluorescence data, and this enhanced information is integrated into a robotic laser ablation system. The accuracy of the fluorescent measurement of the tumor is improved using high-precision spectral analysis. The fluorescence assists in the detection of malignant brain tumors intraoperatively and improves their removal rate.
\end{abstract}

\section{Introduction}

Malignant gliomas are the most common primary brain tumor. During tumor ablation surgery, the surgeon needs to identify the edges of the tumor in order to perform a complete tumor resection. Surgeons can resect most tumors with an accuracy of millimeters using a combination of conventional surgical instruments and a computeraided navigation system with diagnostic images, such as magnetic resonance (MR) and computed tomography (CT) images. However, regardless of the extent of the surgery, some tumor cells remain in the areas adjacent to the tumor removal area. These residual cells, especially those of malignant tumors like glioma, impair the prognosis of the patient. It is necessary to resect the tumor completely while preserving as much normal tissue as possible. 
During neurosurgery, cerebrospinal fluid leakage and surgical interventions deform the brain tissue. This so-called "brain shift" can be as much as several tens of millimeters and continues to increase during the surgical procedure [1]. There is thus a strong need for accurate and precise image-guided surgical navigation based on intraoperative imaging. However, image-guided navigation can be off by as much as few millimeters due to inaccurate registration of the preoperative diagnostic images and intraoperative images [2]. Furthermore, there is a tradeoff between more frequent image acquisition for more accurate navigation and less frequent acquisition for computer resource conservation.

Several advanced surgical tools and imaging techniques have been developed and are becoming widely used to guide surgeons in tumor resection. One technique is to illuminate the brain tumor tissue with 5-aminolevulinic acid (5-ALA)-induced fluorescence. This acid is a natural biochemical precursor of haemoglobin that elicits synthesis and accumulation of fluorescent porphyrins in various epithelia and cancerous tissue [3]. In neurosurgery, surgeons wear a modified neurosurgical microscope and use violet-blue light to see the fluorescent tissue. The fluorescence is used to identify residual malignant glioma intraoperatively so that the surgeon can improve the accuracy of surgical treatment [4-7].

To improve the accuracy of the treatment, we have developed a novel therapy system using 5-ALA fluorescence-enhanced MRI navigation integrated into a microlaser ablation treatment system. The boundary between a tumor and normal tissue is distinguished using 5-ALA-induced protoporphyrin IX (PpIX) fluorescence, and the tumor is accurately ablated with the micro laser. The combination of 5-ALA fluorescence guidance and high-precision spectral analysis makes it possible to improve the treatment system with only pre and intraoperative MR imaging.

\section{Materials and Methods}

\subsection{Intraoperative Identification of Brain Tumors Using 5-ALA}

In fluorescence-guided surgery, the patient drinks a glass of water in which 5-ALA has been dissolved before surgery. 5-ALA turns into PpIX when it is ingested by a living organism. The PpIX tends to accumulate in pathological lesions, so when it is excited by blue light, any brain tumors are easy to spot due to the PpIX emitting red fluorescence (Fig. 1a). The malignant brain tumors can be then be resected with a microscope equipped for fluorescence-guided surgery. The tumor regions are intuitively recognized by the surgeon with the guidance of this system.

In this study, spectra of brain tissues were acquired in vivo, and promising optical filters were selected through simulation using these spectra. In addition, a method was developed for detecting tumor regions using a two-dimensional histogram acquired from two intensity images. The system was evaluated through fluorescent measurement of brain tissues in vivo, and the measurement results were compared with the simulation results. Furthermore, the regions in which the fluorescent properties were uniform were identified by analyzing the image intensity of the fluorescence. We divided the observed tissues into "tumor", "non-tumor" and "blended boundary" using the spectral analysis results. 


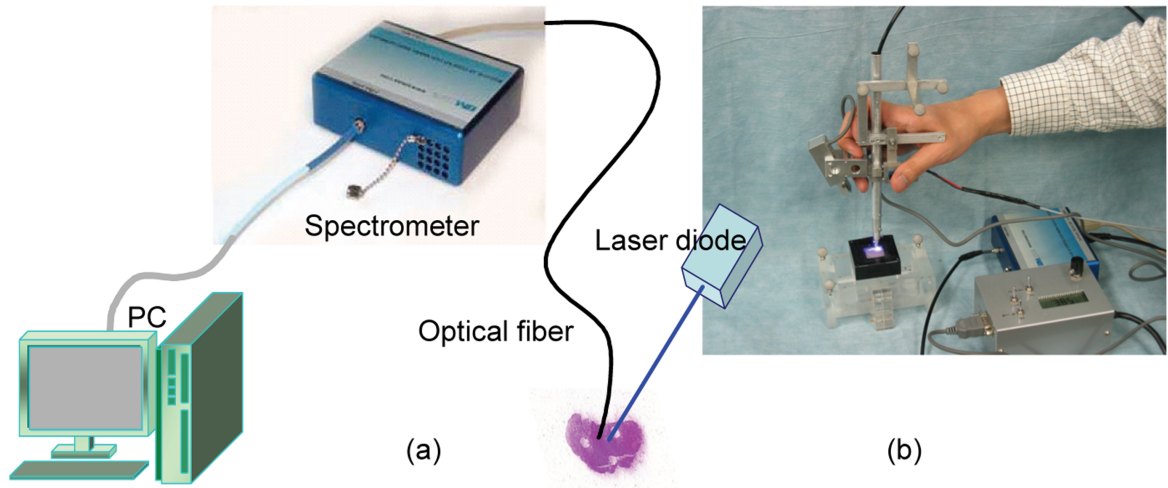

Fig. 1. 5-ALA-induced protoporphyrin IX (PpIX) fluorescence spectral analysis system

A detection probe was designed and used to assess the use of fluorescence in identifying malignant glioma after administration of 5-ALA for collecting PpIX fluorescence (Fig. 1b). The fluorescence was transferred to a spectrophotometer through an optical single-mode fiber for spectral analysis. The detector had a different diameter of 7,5 , or $3 \mathrm{~mm}$, and aspheric lenses were used to correct for aberrations. The detection resolution was set to $0.6 \mathrm{~mm}$ in consideration of the tradeoff between the acquired light intensity (which is in proportional to the square of the detection diameter) and measurement accuracy. A band-pass filter (over $60 \%$ transmission at $635 \mathrm{~nm}$ and up to $5 \%$ at $670 \mathrm{~nm}$ ) was fixed to the tip of the detector. The filter cut off the excitation light and the guide laser light (peak at $670 \mathrm{~nm}$ ) of the autofocus (AF) system. The working distance between the detector and tumor was a maximum of $16 \mathrm{~mm} \pm 0.5 \mathrm{~mm}$.

\subsection{MR Imaging Navigation System}

Removing malignant glioma completely in neurosurgery is a complicated task. Obscure boundaries between normal and abnormal tissues prevent the surgeon from identifying the edges of the glioma accurately. Although the 5-ALA-induced fluorescent-guided system can provide high-precision identification of the tumor surface, information about the whole structure of the tumor, especially in the depth direction, is also needed.

MRI-based navigation helps surgeons to identify the entire tumor in a clinical implementation. Although brain shift occurs during an operation, intraoperative information can be obtained with an image scanner such as an open-MRI device. The intraoperative information acquired by various surgical measurement systems must be integrated for effective surgical navigation. We integrated intraoperative measurement with 5-ALA-induced fluorescence to form an MR imaging navigation. This combined system provides an intraoperative overview of both the information about the tumor surface obtained by fluorescence and the 3-D structure of the tumor obtained by MRI.

\subsection{System Integration}

Our laser ablation system includes a 5-ALA-induced PpIX fluorescence tumor detector, a micro laser module, the MR imaging navigation system, and an automatic 
focusing and robotic scanning system. As shown in Fig. 2, the fluorescence detection probe and micro laser probe are attached to the AF system. The data from the spectral photometer is analyzed using a PC. The analyzed results are used for assisting intraoperative detection of the brain tumor and its boundaries. Switching of the micro laser and scanning with the stepping drive are both controlled by the PC. The surgical field view can be observed using a CCD camera combined with the AF system. The navigation system is used to control electric motors that position the fluorescence measurement and laser ablation instruments. Since the intraoperative information of the surgical area is displayed in this system, the surgeon can treat both the surface and sides of the tumor. The micro laser has a wavelength of $2.8 \mu \mathrm{m}$, which is mostly absorbed by water. The laser is effective only on the surface of brain tissue, enabling precise ablation at the boundary between the tumor and normal tissue.

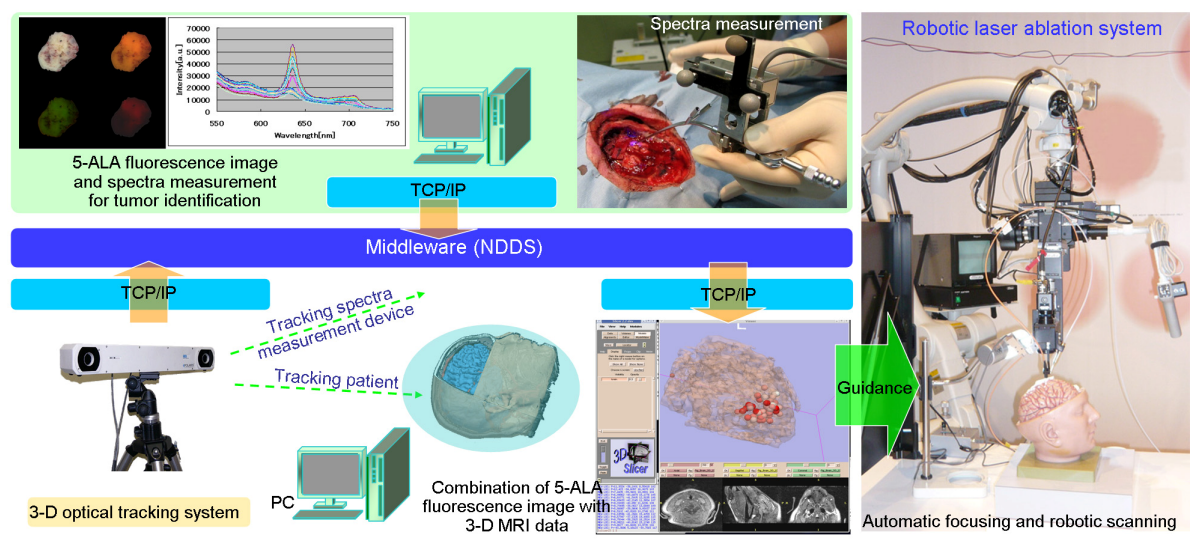

Fig. 2. System configuration of laser ablation treatment with guidance of 5-ALA-enhanced intraoperative navigation

\subsection{Patient-Image Registration}

The MR imaging navigation system is registered using a POLARIS optical tracking device. During the operation, the distance between the ablation laser and tumor can be adjusted using the AF system, which is fixed to the fluorescence spectra acquirement devices. These devices are attached by anther optical tracking probe, which can be tracked by the same optical tracking device. Patient-image registration can be based on a set of distinct features such as geometrical landmarks or natural anatomical landmarks that can be accurately identified in both spaces. The fiducial markers used in image registration provide pairs of corresponding 3-D points in the spaces to be registered. Once the coordinates of the fiducials in the information space and in the patient coordinate space have been determined, the geometric transformation that associates these two spaces can be derived. In this study, a set of MR markers was attached the patient's body, enabling us to calculate the transformation matrix between the original spectra device and then calculate the relationship between the patient's body and the navigation system. Since brain shift leads to large errors with preoperative or even intraoperative MR imaging, we take the intraoperative spectra 
information and register it to the MRI navigation system after finishing rough patientimage registration. The fluorescence information can be integrated into the MRI. The boundary identified by the fluorescence can be used to correct for errors between the MR imaging and the patient's body. Thus, the information provided by the MRI navigation system is enhanced by the addition of fluorescence data.

\section{Experimental Devices and Results}

We combined 5-ALA-induced PpIX fluorescence and MR imaging in the tumor detection system and integrated the system with a micro ablation laser module for treatment. We conducted a set of combination experiments to evaluate the system using a biomedical stimulant material (a phantom) and a porcine brain.

\subsection{Phantom Evaluation Experiment}

We fabricated a container of $122 \mathrm{~mm} \times 70 \mathrm{~mm} \times 49 \mathrm{~mm}$ with a set of POLARIS markers attached along its circumference and placed a phantom $(20 \mathrm{~mm} \times 20 \mathrm{~mm})$ inside it (Fig. $3 a)$. The phantom was an agar plate containing Intralipid-10\%, which is intravenous lipid emulsion used as a scattering medium. The concentration of the Intralipid-10\% was adjusted to obtain an optimal scattering coefficient, one that was close to that of glioma [8]. Half of the phantom contained PpIX, and the other half did not (Fig. 3a, upper right).
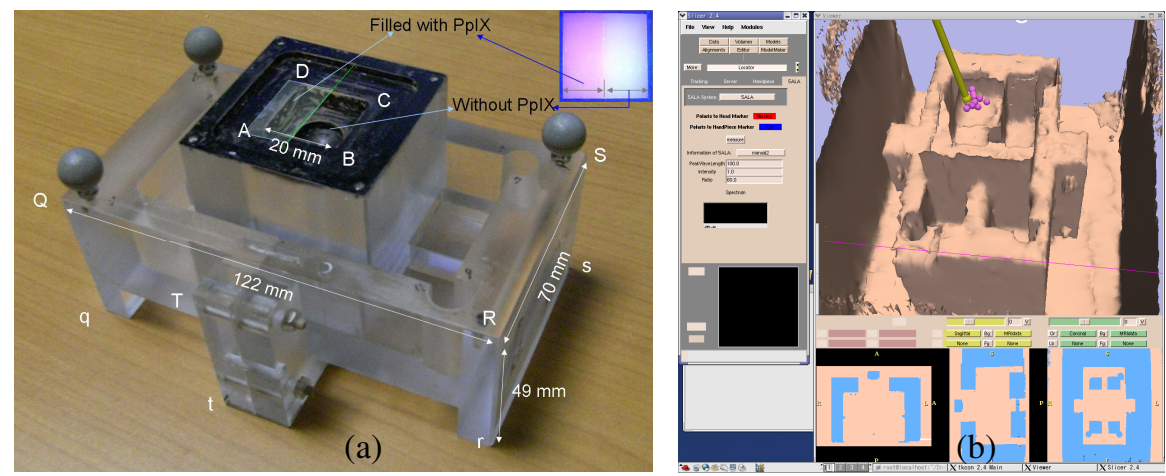

Fig. 3. Phantom for evaluation experiment: (a) container with set of POLARIS markers attached along circumference and PpIX-filled agar phantom; (b) registration of PpIX fluorescence results with 3-D model generated from MRI

The rectangular area in Fig. 3a shows the area scanned the system. The container was filled with water and scanned by MR. The MR image data for the phantom consisted of one set of coronal images with a $1.0 \times 1.0-\mathrm{mm}$ in-plane resolution and a $1.0-\mathrm{mm}$ slice thickness. Five points $(\mathrm{Q}, \mathrm{R}, \mathrm{S}, \mathrm{t}, \mathrm{s})$ were used for image registration within the physical space of the phantom. Four points (A, B, C, D) were used for registration accuracy evaluation. The TRE for 100 tests of the four points was $1.79 \pm 0.35 \mathrm{~mm}$. 
We conducted another set of experiments to evaluate the MRI navigation system. The measured fluorescence spectra were registered on the phantom model generated by MRI (Fig. 3b). The results showed that the system could identify the threedimensional boundary between the area with fluorescence and that without fluorescence and that it could precisely ablate the fluorescent area with automatic scanning.

\subsection{In Vivo Testing of Spectra Measurement and Analysis}

An in vivo experiment with a porcine brain was conducted to evaluate the accuracy of tumor identification (Fig. 4a). The target was the brain surface exposed by craniotomy under anesthesia. Spectra of the brain tissues were acquired in vivo. Promising optical filters were selected through simulation using these spectra. The tumor regions were detected using a two-dimensional histogram acquired from two sets of intensity images. The samples were evaluated through fluorescent measurement of brain tissues in vivo. A threshold was set for the intensity level of the measured fluorescence.

A surgeon or an experimenter determined the threshold of the fluorescence by viewing the measurement area on the CCD camera image. When the scanning was

(a)

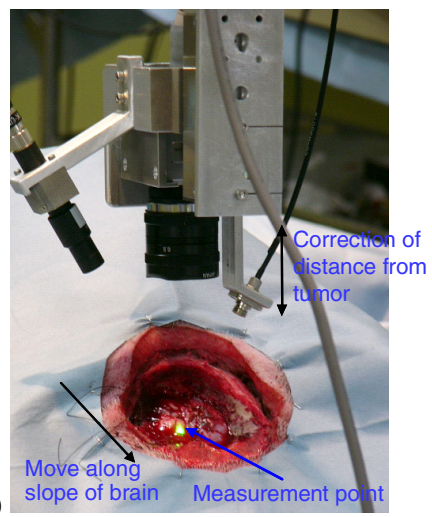

(b)

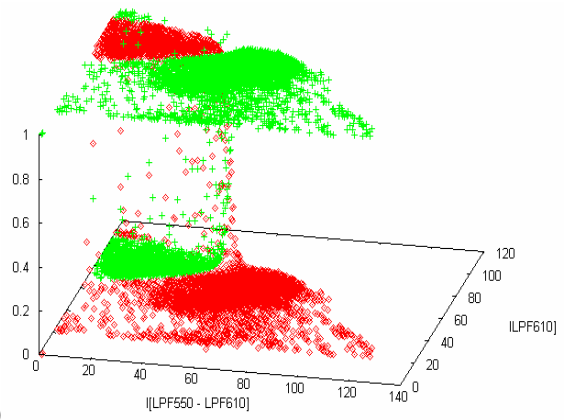

Fig. 4. (a) Experimental device for spectral measurement; (b) results of spectral analysis

(a)

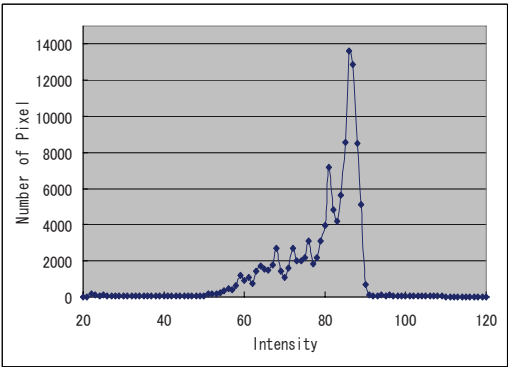

(b)

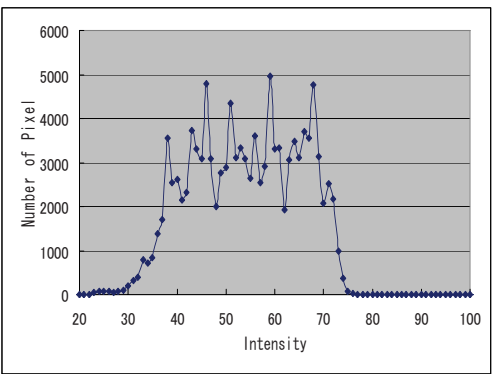

Fig. 5. Results of spectral analysis with (a) LPF of $610 \mathrm{~nm}$; (b) BPF of $636 \mathrm{~nm}$ 
finished, the micro laser was automatically irradiated on the region over the threshold. The results of the measurement were matched with the simulation results. Figure $4 \mathrm{~b}$ shows the probability distributions for the malignant and non malignant glioma, which could be clearly distinguished. Regions in which fluorescent properties were detected were statistically analyzed. Figure 5 shows the results of spectral analysis of the relationship between intensity and pixel number with a low pass filter (LPF) at $610 \mathrm{~nm}$ and a band pass filter (BPF) at $636 \mathrm{~nm}$.

\subsection{Combination of Fluorescence with MRI}

We conducted a clinical trial to evaluate the feasibility of the developed system. MR scanning was performed to image the 3-D structure of a brain (256×256 pixels, 128 slices, 1.0-mm thickness). Volumetric MR images of the brain were reconstructed, and the results were combined with 5-ALA-induced PpIX fluorescence. The images were integrated in neurosurgery and displayed for guidance during laser ablation implementation (Fig. 6a). The weak fluorescence signals in the boundary area were strengthened by supplementation of MR information. The stepping-motor-driven micro laser ablation device was controlled by a PC, and the tumor was photocoagulated by a scanning laser (Fig. 6b).

(a)

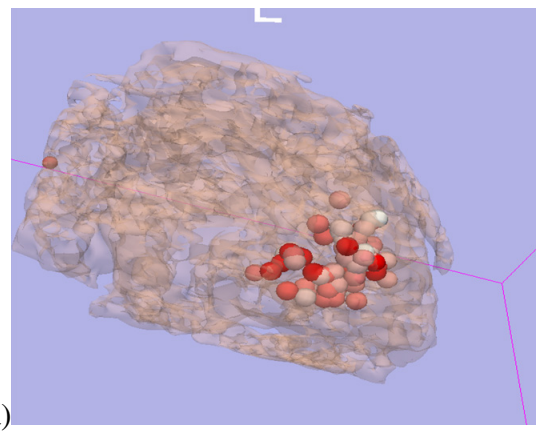

(b)

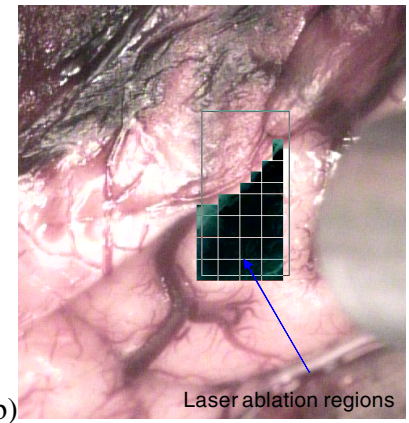

Fig. 6. (a) Combination of fluorescence spectra with MRI; (b) ablation region for laser scanning

\section{Discussion and Conclusions}

We developed a computer-aided navigation system using 5-ALA-induced PpIX fluorescence and 3-D MRI anatomical structure data for robotic laser ablation of tumor. The ablation system has automatic focusing and a robotic scanning mechanism and was designed for malignant glioma treatment. Ablation is performed using fluorescence information with robotic position control.

The combination of fluorescence measurement and navigation system registration was tested using a biomedical stimulant phantom and a porcine brain. The measurement areas for both were separated into fluorescent and non-fluorescent parts. The results showed that the system can identify the regions with fluorescence appropriately from the measurement data and that the ablation system can be automatically guided by the navigation system. 
The boundaries between tumors and normal tissues are often unclear, and parts of a tumor can extend into normal tissue. Therefore, deciding the threshold for the fluorescence data and identifying the area to be ablated are challenging. We are thus developing a system with improved measurement accuracy that can better handle complicated tumor tissue structures. To enable the combined tumor region to be automatically discriminated by using fluorescence spectra, we will investigate a multiple classification analysis based on other spectral features as well as the peak intensity of the fluorescence. This system will also combine automatic processing with robotic laser ablation.

\section{Acknowledgment}

This work was supported in part by Grant for Translational Systems Biology and Medicine Initiative (TSBMI) from the Ministry of Education, Culture, Sports, Science and Technology of Japan. H. Liao was also supported by Grant for Industrial Technology Research of New Energy and Industrial Technology Development Organization, Japan, and Grant-in-Aid for Scientific Research of the Japan Society for the Promotion of Science.

\section{References}

1. Clatz, O., Delingette, H., et al.: Robust nonrigid registration to capture brain shift from intraoperative MRI. IEEE Trans. on Medical Imaging 24(11), 1417-1427 (2005)

2. Gholipour, A., Kehtarnavaz, N., et al.: Brain Functional Localization: A Survey of Image Registration Techniques. IEEE Trans. Med. Imag. 26(4), 427-451 (2007)

3. Regula, J., MacRobert, A.J., Gorchein, A., et al.: Photosensitisation and photodynamic therapy of oesophageal, duodenal, and colorectal tumours using 5-aminolaevulinic acid induced protoporphyrin IX - a pilot study, Gut, vol. 36, pp. 67-75 (1995)

4. Stummer, W., Novotny, A., Stepp, H., et al.: Fluorescence-guided resection of glioblastoma multiforme by using 5-aminolevulinic acid-induced porphyrins: a prospective study in 52 consecutive patients. J. Neurosurg. 93, 1003-1013 (2000)

5. Stummer, W., Stocker, S., Wagner, S., et al.: Intraoperative detection of malignant gliomas by 5 -aminolevulinic acid-induced porphyrin fluorescence. Neurosurgery 42, 518-525 (1998)

6. Noguchi, M., Aoki, E., et al.: A Novel Robotic Laser Ablation System for Precision Neurosurgery with Intraoperative 5-ALA-Induced PpIX Fluorescence Detection. In: Larsen, R., Nielsen, M., Sporring, J. (eds.) MICCAI 2006. LNCS, vol. 4190, pp. 543-550. Springer, Heidelberg (2006)

7. Maruyama, T., et al.: Intraoperative detection of malignant gliomas using 5-Aminolevulinic acid induced protoporphyrin fluorescence, open MRI and real-time navigation system. Computer Assisted Radiology and Surgery 15, 270-275 (2001)

8. van Staveren, J., Moes, J.M., van Marle, J., Prahl, A., van Germert, J.C.: Light scattering in Intralipid-10\% in the wavelength range of 400-1100 nm. Appl. Opt. 30, 4507-4514 (1991) 